\title{
Diseño de información para el web, 1996-2000. Un análisis bibliográfico selectivo
}

\author{
Por Jesús Tramullas
}

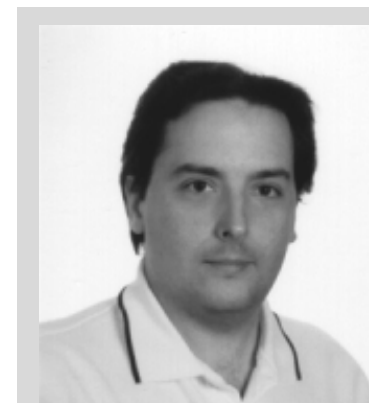

Jesús Tramullas

Resumen: Este trabajo revisa la evolución del diseño de información para el world wide web, durante el período 1996-2000. Se realiza un análisis de los principales y más conocidos manuales publicados sobre la cuestión, así como los enfoques y aportaciones de los mismos. El estudio permite establecer la existencia de varias corrientes y escuelas.

Palabras clave: Diseño de información, Diseño web, Arquitectura de la información.

Title: Information design for the web, 1996-2000. A selected bibliographical analysis

Abstract: This paper reviews developments in information design between 1996-2000, in the context of the World Wide Web. An analysis is made of the major, best known manuals and books on this subject, highlighting the focus and main features of each. The study reveals different tendencies and schools of thought on this subject.

Keywords: Information design, Web design, Information architecture.

Tramullas, Jesús. "Diseño de información para el web, 1996-2000. Un análisis bibliográfico selectivo". En: El profesional de la información, 2001, diciembre, v. 10, n. 12, pp. 34-40.
\end{abstract}

\section{Planteamiento}

El diseño de información para el web es una disciplina en auge y en constante desarrollo. La novedad del medio, unida a su interactividad y a la acelerada aparición de nuevas técnicas y herramientas, ha permitido constituir un campo nuevo en el que se han integrado progresivamente profesionales y expertos provenientes de muy diversas áreas, cada uno de los cuales ha aportado aproximaciones y métodos que lo han enriquecido. El objetivo de este trabajo es identificar, mediante el análisis de las monografías más interesantes, las líneas y enfoques que se han aplicado en el mismo, incidiendo en las aportaciones que han realizado para ello.

\section{«La conceptualización y defini- ción de la disciplina 'diseño de información' resulta compleja por las múltiples facetas que se integran en la misma»}

Se han seleccionado como puntos de referencia las monografías y manuales más reconocidos, ordenados cronológicamente ya que por las características del medio al que se dirigen, son los que reúnen los requisitos necesarios para satisfacer el propósito del estudio. Esta decisión nos ha llevado a no incluir material publicado en revistas y congresos especializados ya que, aunque suelen ofrecer las garantías de calidad y novedad exigibles, en realidad no tienen el mismo impacto sobre la actividad profesional que la obtenida por las obras referenciadas.

Cabe señalar, sin embargo, que casi todas ellas tienen detrás un fuerte bagaje científico, identificable en la actividad de los correspondientes autores. Como puede deducirse de este planteamiento, tampoco se han incluido guías o manuales dedicados a lenguajes de etiquetado o de programación para el www dado que no están dedicados al diseño o arquitectura de información para el web.

Se ha seleccionado un período de cinco años, el comprendido entre 1996 y 2000 , ya que durante el mismo puede apreciarse el surgimiento y desarrollo de varios enfoques o líneas de trabajo sobre el tema en cuestión. En realidad, es difícil encontrar otro material previo en el cual se aborde el problema de diseño de información para el web, aunque sí pueden hallarse obras de referencia obligada sobre el tema para aplicaciones en entorno informático ${ }^{1}$. Por otra parte, la conceptualización y definición de la disciplina "diseño de información" resulta compleja por las múltiples facetas que se integran en la misma, por lo que remitimos a las referencias básicas para comprender sus características $^{2}$. Para terminar este planteamiento, desde nuestra perspectiva el proceso de diseño y creación de documentos digitales $^{3}$ es inherente al desarrollo actual de las ciencias de la información y la documentación, por lo que es en este marco donde debe entenderse el diseño de información, como ya ha sido aceptado por asociaciones de referencia como Asist.

\section{Análisis bibliográfico}

-Sano, Darrel. Designing large-scale websites. New York: John Wiley, 1996.

El libro de Sano (en ese momento en la Netscape Communications Corporation) tuvo la virtud de introducir una primera aproximación al rigor de la metodología de desarrollo de productos de información en el mundo web. Hasta ese momento los manuales y trabajos al uso se centraban en explicar las características de 


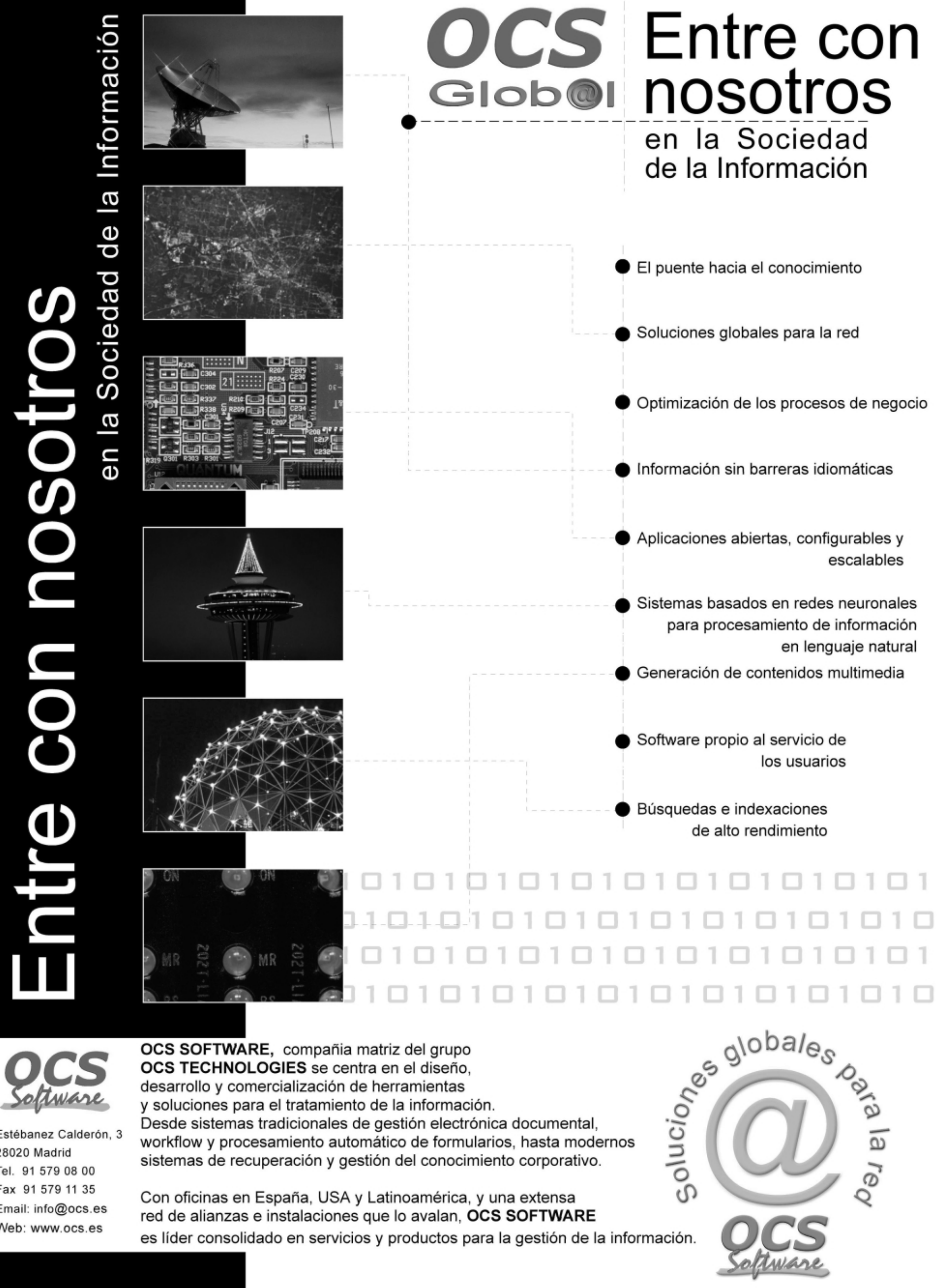


html como herramienta de creación de documentos hipertextuales, las prestaciones de los lenguajes de etiquetado y las posibilidades de integración de multimedia e interacción mediante cgis. El autor estableció una primera diferenciación según el objetivo de las sedes web, para pasar a insistir en la importancia del marco organizativo al que servía, la estructura del espacio informativo y los tipos de páginas que iban a tener, que, en realidad, correspondían a las clases de contenido informativo y de interacción que podían ofrecer las mismas.

Otra de las aportaciones fue la adopción de lo que el autor definió como un método de diseño visual para el web (capítulo 4), fundamentado en la incorporación en los documentos de componentes gráficos que indicaban las relaciones visuales entre ellos, así como el contexto informativo en el cual adquirían su sentido, merced a la adopción de elementos simples y consistentes que permitían identificar al usuario dónde se encontraba y qué estaba haciendo dentro del espacio informativo de la sede web.

-Powell, Thomas A.; Jones, D. L.; Cutts, D. C. Web site engineering. Beyond web page design. Upper Saddle River: Prentice Hall, 1998.

El prefacio de esta monografía incluye la frase clave que debería haber presidido el diseño de información para el web desde sus comienzos: "web site design is not graphic design" (pág. ix). El contenido posterior insiste, de una manera más rigurosa y sistemática, en la adopción de un enfoque de ingeniería del software para la producción de sedes web, pero modificado teniendo en cuenta que "web engineering is not software engineering" (p. 47). Si bien el método ofrece las fases clásicas de un modelo de cascada, los autores proponen su modificación a espiral, para hacerlo más acorde con las particularidades del web.

Es interesante indicar que el enfoque toma como puntos de partida el estudio del web como medio de comunicación, no como aplicación software, y el análisis de la actividad del usuario durante una sesión. Esto conlleva que el proyecto comience por la definición del problema y por la obtención de la solución al mismo, dedicando considerables recursos a la especificación de requerimientos. Hay que destacar que el capítulo 6 en su totalidad trata temas como el diseño de información, la diferencia entre aplicación e información, el diseño de navegación, etc., mientras que el ca- pítulo 8 se dedica a las pruebas y test de funcionalidad, contenido y usuarios.

\section{«El proceso de creación de do- cumentos digitales es inheren- te al desarrollo actual de las ciencias de la información y la documentación, por lo que en este marco debe entenderse el diseño de información»}

Otro de los valores de este trabajo es considerar que la publicación en web va a aumentar considerablemente en virtud de la generación dinámica de documentos desde bases de datos, así como que esta creciente problemática de las herramientas de diseño y publicación necesitará metodologías complejas. La evolución durante los dos últimos años del software de gestión de contenidos ha sido buena muestra de ello ya que ha combinado las características del desarrollo estructurado con los sistemas de publicación dinámica de contenidos.

-Fleming, Jessica. Web navigation. Designing the user experience. Sebastopol: O’Reilly, 1998.

En 1998 la editorial O'Reilly publica dos libros clave en el diseño de información para el web. La obra de Fleming incide en el estudio de la experiencia del usuario, que se desarrolla a través de las interfaces. Desde esta perspectiva introduce el enfoque de "user-centered design", que se acomoda en el proceso de interacción hombre-máquina y que tiene lugar de manera fundamental en el mecanismo de navegación que lleva a cabo el usuario mientras busca la información necesaria para alcanzar sus objetivos. Aunque aborda las cuestiones de proceso de diseño y arquitectura de la información, lo realmente interesante es el estudio que hace sobre las características de la implementación de la navegación en los diferentes tipos de sedes. Identifica los objetivos específicos de los usuarios en cada tipo de sede y, conforme a ello, propone una organización tanto de contenidos como de navegación inherente diseñada para satisfacer esos propósitos.

Los estudios de caso, las entrevistas a diseñadores que enfocan su trabajo en la experiencia de usuario, así como la recopilación de lecturas y enlaces complementarios son un valor añadido para esta obra que es un claro exponente de esta corriente. 


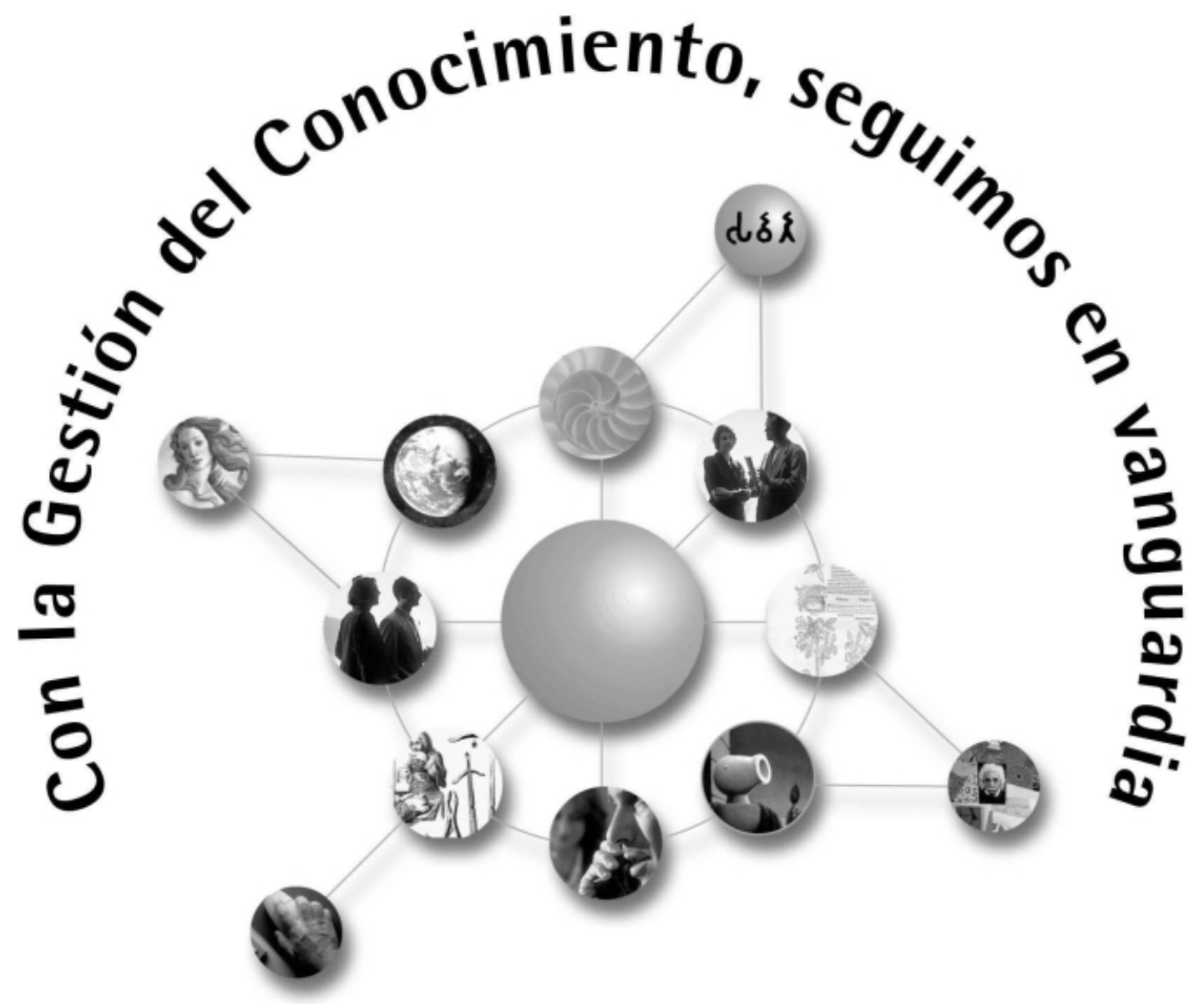

\section{Estamos certificados
en calidad ISO 9002}

Nuestras soluciones

Gestión del conocimiento Inmagic DB/Textworks Webpublisher Spider

\section{Bibliotecas \\ Aurora \\ Documarc \\ Glas \\ Tot}

Archivos

Anser por Bureau Veritas Quality International en:

- Consultores en recursos de información

- Gestión del conocimiento

- Intranet/Extranet/Internet

Edición electrónica

- Suscripciones a bases de datos y a revistas electrónicas (http://informame.doc6.es)

Programas de gestión documental

Cursos de formación 
- Rosenfeld, Louis; Morville, P. Information architecture for the world wide web. Sebastopol: O'Reilly, 1998.

Este manual ha alcanzado la categoría de clásico en el campo de la arquitectura de la información para la web, hasta tal punto que muchas de las obras posteriores incluyen estructuras y enfoques similares en lo concerniente al tema. Ambos autores se han formado en el campo lis (library and information studies) y su concepto nuclear es la arquitectura de la información, que definen como "la ciencia de la organización de la información", construyendo para ella un fundamento teórico en los capítulos 2 y 3 . Como se señala en el prefacio, el libro es útil para "anyone who confuses web page design with web site design" (pág. xv).

En la metódica organización del texto establecen, de manera indirecta, cuatro grandes bloques de actividad correspondientes a: la organización de la información, el diseño de los sistemas de navegación, los procedimientos de etiquetado e identificación de contenidos y los sistemas de búsqueda de información (en el contexto interno de la sede). La influencia de la tendencia lis se aprecia, por ejemplo, en la propuesta de utilización de clasificaciones científicamente construidas para los sistemas de etiquetado de contenidos, o en las técnicas de estructuración de nodos informativos. La plasmación de los principios teóricos se muestra en los capítulos 7,8 y 9 dedicados a un método completo de diseño y creación de sedes web.

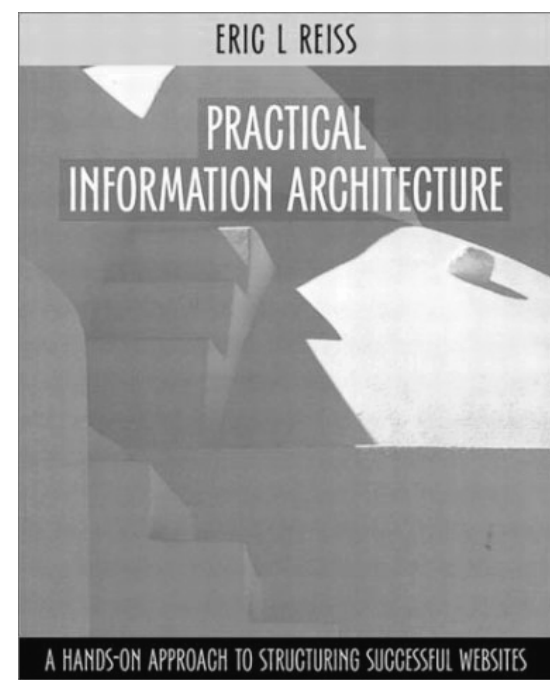

zación y desarrollo del equipo humano de trabajo. A ello dedica dos capítulos completos así como partes componentes de otros tres, a lo que hay que unir la comprensión de las necesidades y objetivos del cliente, frente a un único capítulo dedicado al proceso de desarrollo.

\section{«El software de gestión de con- tenidos ha combinado las ca- racterísticas del desarrollo es- tructurado con los sistemas de publicación dinámica de conte- nidos»}

—Lynch, Patrick J.; Horton, S. Web style guide. Basic design principles for creating web sites. New Haven: Yale University Press, 1999.

Este afamado manual es la plasmación en papel de la guía de diseño de ambos autores disponible en el web desde 1997. Ofrece una perspectiva integradora que combina los enfoques de arquitectura de la información con las interfaces de usuario junto con una realización equilibrada y consistente de las páginas. Especialmente interesante por sus ejemplos y propuestas es el capítulo 4 dedicado al diseño de páginas, ofreciendo un estado del arte sobre el tema de plena vigencia, sin rehusar entrar en cuestiones técnicas que afectan a las características de presentación y disposición de los documentos, todo ello muy influenciado por la perspectiva de la creación y pro-

-Burdman, Jessica. Collaborative web development. Strategies and best practices for web teams. Reading: Addison-Wesley, 1999.

Burdman ha insistido en la importancia que, junto a una adecuada metodología, tiene la selección, composición y organización del equipo de trabajo necesario para crear y mantener una sede web. La autora tiene el acierto de señalar que no existe ni puede existir un único método para ello, ya que el contexto de objetivos, necesidades y equipo de trabajo en cada caso puede variar notablemente. Compara en una interesante tabla las características del web, del software y de la publicidad, así como la influencia de cada una de ellas en el diseño (pp. 3-5).

Debe destacarse que, desde la perspectiva de la autora, además de la relatividad que adquiere el proceso de diseño (insistiendo en la existencia de buenas prácticas comunes que deben adecuarse a cada situación), la cuestión clave del éxito es la composición, organi- ducción técnica de documentos. Se trata de una recopilación ordenada y metódica de "buenos usos y costumbres", dentro del marco de un proceso general de diseño, por lo que mantiene gran parte de su validez.

http://info.med.yale.edu/caim/manual

-Spool, Jared; Scanlon, T.; Schroeder, W.; Snyder, C.; Deangelo, T. Web site usability. A designer's guide. San Francisco: Morgan Kaufmann, 1999.

El trabajo de estos autores, contra lo que puede parecer por el título de la obra, no es una guía para diseñadores. Por el contrario, se trata de las conclusiones alcanzadas tras someter, durante 1997, a un conjunto seleccionado de sedes web a un análisis de "usability" tan de moda en la actualidad. Este estudio se llevó a cabo usando como marco un conjunto de formularios cuyo examen permite extraer conclusiones sobre cómo los usuarios utilizan las fuentes y la información puestas a su disposición. Sin embargo, los autores estable- 
cen un principio que se ha hecho común: "[...] we probably weren't using these sites in the way that their designers intended" (pág. xv).

El método seguido lo ha convertido en una referencia muy citada, aunque en realidad es un conjunto de mediciones cualitativas basadas en el sentido común. De especial interés es el capítulo 1 en cuanto establece, gracias a los análisis realizados, cinco principios básicos a considerar para el diseño de sedes web: "1. Graphic design neither helps nor hurts; 2. Text links are vital; 3. Navigation and content are inseparable; 4. Information retrieval is different than surfing; 5 . Web sites aren't like software" (pp. 9-13).

-Lowe, David; Hall, W. Hypermedia \& the web. An engineering approach. Chichester: John Wiley, 1999.

El título de esta monografía no debe llevar a pensar que se trata de otro exponente del enfoque de ingeniería del software aplicada a la web. Se trata de un punto de vista que considera las sedes web como aplicaciones hipertextuales e hipermediales. Fundamentado de manera rigurosa, analiza en primer lugar el concepto de hipermedia así como los modelos de información susceptibles de uso y las características de las aplicaciones de este tipo, para proponer una actividad de diseño, creación y mantenimiento a la que denominan "hypermedia engineering", que tiene tanto en la calidad del producto como en la del proceso un componente esencial.

En lo que respecta al diseño de información, hay que reseñar el exhaustivo método de construcción de sedes web que se articula en la segunda parte del libro (capítulos 7, 8 y 9). Organizado según procesos, métodos y técnicas, ofrece un completo marco de desarrollo que revisa las posibles opciones y variedades existentes de tal forma que estudia modelos de producto, de proceso, documentación del proyecto, técnicas de estructuración de la información, establecimiento de enlaces, navegación, etc. Para terminar, analiza detenidamente varios productos y metodologías de diseño y desarrollo de aplicaciones hipermediales, concluyendo con unos útiles apéndices que sirven como guía de partida para crear y producir una aplicación hipermedia.

-Pearrow, Mark. Web site usability handbook. Rockland: Charles Media River, 2000.
Frente al contenido empírico del libro de Spool, esta obra es un verdadero manual de análisis y técnicas de "usability" aplicadas tanto a estudios de sedes como a los procesos de diseño, muy superior, por ejemplo, al afamado libro de Nielsen. Su base teórica es el diseño centrado en el usuario ("user-centered design"), al cual dedica el capítulo 2 al completo, así como los factores humanos que intervienen. Desde estos fundamentos establece el estudio como un proceso continuo de interacción entre producto, objetivo y actividades de usuario final. Analiza la evaluación heurística aplicada al web, una herramienta clave para la usabilidad, así como el establecimiento de laboratorios especializados y la confección de test experimentales.

Desde esta perspectiva, y al igual que otros textos enfocados a la usabilidad, su tratamiento del diseño de información es limitado, fijándose en aspectos esencialmente visuales del mismo.

—Nielsen, Jakob. Designing web usability. Indianapolis: New Riders, 2000.

Resulta obligatorio incluir el libro de Nielsen en cuanto a su impacto, ya que ha atraído la atención de muchos diseñadores sobre la importancia de la "usability". Sin embargo, no es una obra sobre diseño ni de páginas ni de sedes. Se trata de una recopilación de consejos y pensamientos, muchos de los cuales ya habían sido publicados con anterioridad en la sede web del autor.

\section{http://www.useit.com}

-Reiss, Eric L. Practical information architecture. A hands-on approach to structuring successful websites. Harlow: Addison-Wesley, 2000.

La arquitectura de la información tiene en este manual otra referencia obligada en cuanto que es un desarrollo profundo de las líneas y métodos presentados por Rosenfeld y Morville. La propuesta comienza con un planteamiento del escenario de actuación, tareas a desarrollar, definición de contenidos (por extensión, del tipo de sede), así como identificación y provisión de servicios útiles para el usuario. Tras este inicio, el autor dedica la segunda y la tercera parte del libro (en total doce capítulos) al diseño de la estructura (o arquitectura) de información de la sede. Se estudian los diferentes niveles de profundidad, la utilización de enlaces en la arquitectura y la importancia de lo que llama "secondary features", en los que incluye mapas e índices de sedes, faqs, motores de búsqueda interna, mecanismos de feedback con el usuario, etc. La evolu- 
ción de los productos informativos en el web se aprecia en la importancia que adquiere la construcción de subsedes, a la que se dedica un capítulo propio.

\section{Enfoques y líneas en el diseño de información para el web}

La revisión llevada a cabo permite identificar un conjunto de rasgos comunes que delinean una aproximación empírica a la noción de diseño de información para el web. En primer lugar se reconoce la diferencia que supone este medio frente a otros sistemas y entornos de producción de documentos. Por otra parte se establece, como una característica intrínseca a estos sistemas de documentos, la existencia de un usuario final que interactúa de forma dinámica con el contenido informativo y con servicios de valor añadido mediante una interfaz dedicada a ello. En tercer lugar, se reconoce este proceso como informativo, basado en documentos digitales. Por último, se indica la necesidad de desarrollar una aproximación metodológica rigurosa para alcanzar los objetivos fijados. Este marco de coincidencia permite establecer la disciplina en cuanto que posee objetivos, campo y materia de aplicación, así como propuestas metodológicas consecuentes y razonadas, perfectamente aplicables en el ámbito que se estudia.

\section{«Burdman ha insistido en la importancia que junto a una adecuada metodología tiene la selección, composición y orga- nización del equipo de trabajo necesario para crear y mante- ner una sede web»}

El análisis también permite delimitar varios enfoques y líneas de actuación de los especialistas puesto que se detectan rasgos definitorios que particularizan a las mismas. En un primer grupo podemos situar a la arquitectura de la información, cuyos máximos exponentes son Rosenfeld, Morville y Reiss. Se caracterizan por el énfasis puesto en la organización y estructuración del contenido informativo como fundamento básico del diseño de información, sin minusvalorar por ello otros componentes del proceso de diseño y del producto final.

Un segundo grupo se corresponde al que tiene como fundamento el diseño centrado en el usuario, que suele orientarse tanto a la creación de interfaces de usuario como a la interacción correspondiente entre hombre y producto informativo. Cabe situar aquí a Sano pero especialmente a Fleming. Debe tenerse en cuenta que el diseño de interfaces de usuario es una disciplina con gran desarrollo en el ámbito anglosajón, que ha influido enormemente en el diseño de información para entornos digitales. A este grupo cabría añadir gran parte del trabajo de Pearrow, aunque se engloba bajo la etiqueta de la "usability". Esta cuestión sirve para introducir el enfoque de usabilidad, que en realidad debe verse como un conjunto de técnicas que aseguren la calidad del producto, aplicables sobre todo el proceso más que como un verdadero método de diseño de información para el web. En este grupo cabe situar a Pearrow, Spool y, por supuesto, a Nielsen.

El cuarto grupo es el conformado por autores provenientes del campo de la ingeniería del software, como Lowe, Hall y Powell. Aportan el punto de vista de ingeniería del software como método de diseño y desarrollo, aunque modificado para adecuarse a las características propias del entorno web y de sus usuarios. Por último, cabe señalar el enfoque sociológico orientado a los grupos de desarrollo, introducido también por Sano y del que Burdman es el exponente principal. Esta tendencia ha sido particularmente interesante en aspectos relacionados con la gestión del conocimiento en internet e intranets y con la adopción de tecnologías web en el contexto de las organizaciones, lo que supera ampliamente los límites de este trabajo. La interacción entre enfoques y el desarrollo de nuevos métodos y técnicas, unidos a la expansión y consolidación de los sistemas de gestión de contenidos, ha cristalizado, en el periodo cronológico estudiado, como un ámbito estratégico para el futuro de la información y la documentación.

\section{Notas}

1. Véase por ejemplo: Horton, W. K. Designing and writing online documentation. New York: Wiley, 1994.

2. Jacobson, R. (ed.). Information design. Cambridge: MIT Press, 1999; Orna, E.; Stevens, G. "Information design and information science: a new alliance?". En: Journal of information science, 1991, 17, 4, pp. 197208; Tramullas, J. "Planteamiento y componentes de la disciplina information design". López Yepes, J. (ed.). I Congreso universitario de ciencias de la documentación. Teoría, historia y metodología de las ciencias de la documentación, 2000, pp. 723-730.

3. Véase la obra de referencia en el campo del diseño de documentos tanto tradicionales como interactivos: Schriver, K. Dynamics in document design. New York: Wiley, 1997. Más información sobre los fundamentos teóricos en:

http://tramullas.com/infodesign/

4. American Society for Information Science and Technology.

http://www.asis.org

Jesús Tramullas, Departamento de Ciencias de la documentación, Universidad de Zaragoza.

tramullas@posta.unizar.es

http://tramullas.com 